\title{
Single ultrafast diffusive conduction based optoelectronic switch for multi-channel operation
}

\author{
Fatih Hakan Koklu and Hilmi Volkan Demir \\ Bilkent Nanotechnology Research Center, Bilkent University, Bilkent, Ankara TR-06800, Turkey \\ Tel. [+90] (312)290-1021, volkan@bilkent.edu.tr \\ Micah Yairi, James S. Harris, Jr., and David A. B. Miller \\ E.L. Ginzton Laboratory and Solid State and Photonics Laboratory, Stanford University, Stanford, CA 94305, USA
}

\begin{abstract}
For multi-channel optical switching, we report single ultrafast diffusive conduction based optoelectronic switches that accommodate $>100$ optical channels (with $2,000 \mathrm{~mm}^{-2}$ channel density and $<10 \%$ crosstalk), on $300 \mu \mathrm{m} \times 300 \mu \mathrm{m}$ devices with switching bandwidths of $>50 \mathrm{GHz}$.

Ultrafast all-optical switching has been extensively investigated and different types of photonic switches have been successfully demonstrated [1-6]. Such optical switches hold great promise for a wide range of high-speed applications that extend from optical wavelength conversion [1] to optical regeneration [2]. Such applications typically require arrays of switches, where a large number of optical channels need to be brought together into a small volume and switched within such limited space [3]. However, it is technically challenging to incorporate a large number of switches into an array integrated on a chip scale and to meet the requirements of such demanding multi-channel applications. In this paper for the first time, for multi-channel applications, we introduce a single ultrafast diffusive conduction based optoelectronic switch that accommodates $>100$ optical channels with a channel density of 2,000 channels per $1 \mathrm{~mm}^{2}$ of lateral device area and $<10 \%$ crosstalk between channels, on a typical $300 \mu \mathrm{m} \times 300 \mu \mathrm{m}$ device with a switching bandwidth of $>50 \mathrm{GHz}$ and a possible aggregate transmission rate of $5 \mathrm{~Tb} / \mathrm{s}$ in principle. Fig.1(a) shows a device schematic of such an ultrafast optoelectronic switch that relies on diffusive conduction and Fig.1(b) depicts a top view micrograph of such a fabricated device [4]. In Fig.1(a), as an exemplary case, four parallel optical channels are shown to be simultaneously incident on a single optoelectronic switch, where each output optical channel is switched independent of the others with the control of the associated input optical channel. Here, each input optical channel optically induces a local voltage change due to the voltage screening of its photogenerated carriers in its vicinity, which changes the absorption level through the switch for the output optical channel; this voltage change, in turn, quickly diffuses away via diffusive conduction across the lateral plane of the switch based on the internal local device RC time constant [4].
\end{abstract}

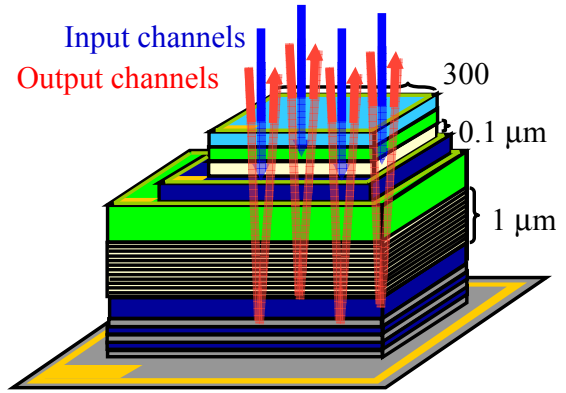

(a)

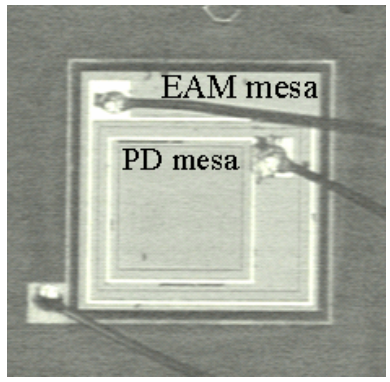

(b)

Fig.1: (a) A device schematic with four input and output parallel channels, (b) a top view micrograph of a fabricated switch.

For multi-channel operation, our approach uses a single optoelectronic switch, which is completely opposite to the traditional idea of arraying many switches. With that, this scheme eliminates the need for switch integration as well as the need for the precise alignment of the integrated individual switches or the optical channels. In this technique, the accommodation of multiple channels on a single switch is possible due to the unique, local behavior of diffusive conduction. In such diffusive conduction based optoelectronic switches, only a small portion of the device dictated by the spot size of the optical channel is switched and, thus, many optical channels can run in parallel normal to a switch as long as they are sufficiently far away from each other. Here, we extend the analysis of the experimental switch demonstration to multi-channel operation, including Green's function diffusive conduction solution and crosstalk calculations. The Green's function solution for the diffusive conduction of an arbitrary, optically induced, local voltage profile of $f(x, y)$ is derived as

$V(x, y, t)=\int_{-\infty-\infty}^{\infty} \int_{-\infty}^{\infty} d x^{\prime} d y^{\prime} f\left(x^{\prime}, y^{\prime}\right) \cdot G\left(x-x^{\prime}, y-y^{\prime}, t\right)$, where $G(x, y, t)=\frac{1}{4 \pi D t} \exp \left(-\frac{x^{2}+y^{2}}{4 D t}\right)$ and $V(x, y, t=0)=f(x, y)$ 
Here $G(x, y, t)$ is the characteristic diffusive conduction decay with $D=1 / R C$ per unit area, which is engineered for high speed operation, for example, about $10 \mu \mathrm{m}^{2} / \mathrm{ps}$ for a $50 \mathrm{GHz}$ switching bandwidth. Fig.2(a) sketches the threelayer RC planes of the switch shown in Fig.1 and Fig.2(b) shows its experimental switching operation for a $50 \mathrm{GHz}$ burst logic pulse train with 20ps repetition period, plotted along with the theoretical simulation of the device based on the diffusive conduction solution. There is a strong agreement between the experimental data and the diffusive conduction based model for a single optical channel operation [4].

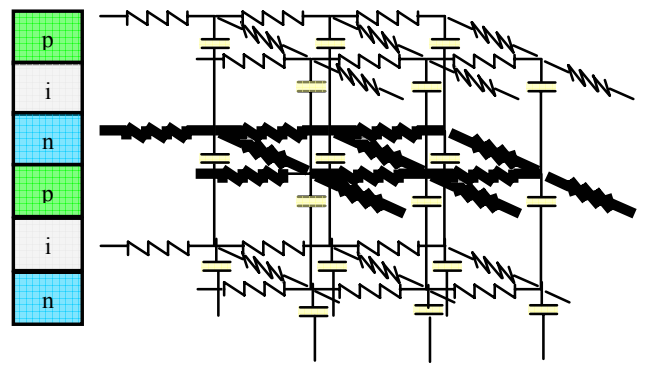

(a)

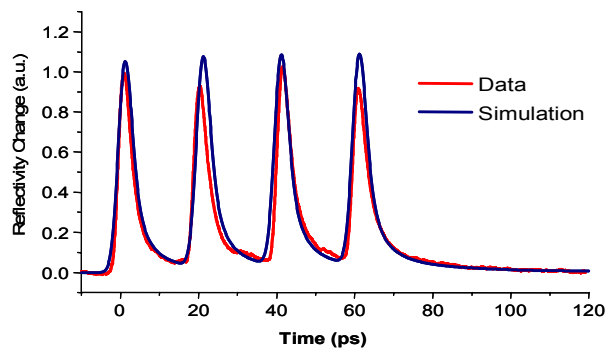

(b)

Fig.2: (a) A sketch of the RC planes of the diffusive conduction switch, (b) the experimental and theoretical switching operation for a single optical channel at $50 \mathrm{GHz}$.

Fig. 3 shows the multi-channel operation of the same diffusive conduction based optoelectronic switch as a function of channel spacing (and channel density) for different device $D$ constants (in $\mu \mathrm{m}^{2} / \mathrm{ps}$ ). We demonstrate that the crosstalk between the parallel optical channels increases with the decreasing channel spacing (and the increasing channel density) as well as with the increasing switch speed. Considering the worst case for the crosstalk calculations for the switches with high extinction ratio $(10 \mathrm{~dB})$, we observe that it is possible to establish multiple optical channels with a density of $2 \mu \mathrm{m}^{-2}$ for $D=10 \mu \mathrm{m}^{2} / \mathrm{ps}$ yielding $<10 \%$ crosstalk between the channels. This makes $>100$ optical channels on a $300 \mu \mathrm{m} \times 300 \mu \mathrm{m}$ switch device with a switching bandwidth of $50 \mathrm{GHz}$ as demonstrated in Fig.2(b).

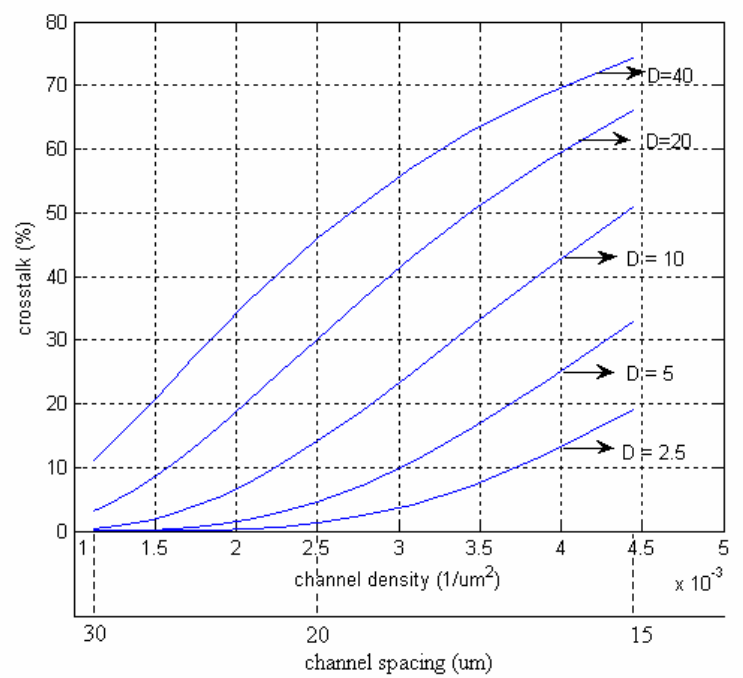

Fig.3: Multi-channel operation: plot of the crosstalk as a function of channel spacing (and channel density) for different device $D$ constants (in $\mu \mathrm{m}^{2} / \mathrm{ps}$ ).

In conclusion, a diffusive conduction based optoelectronic switch proves to be promising for multi-channel operation on a single device as a unique device architecture that possibly avoids device arraying.

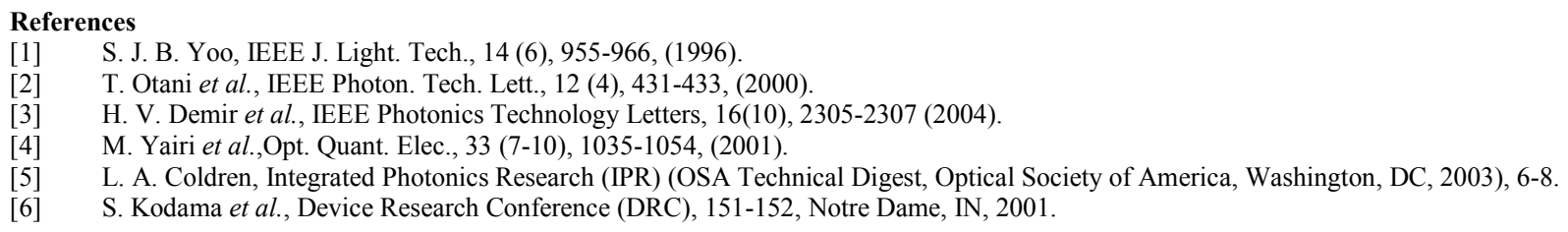

